\title{
The \#NYerORCoverChallenge: What it means for women in cardiothoracic surgery
}

\author{
Mara B. Antonoff, MD, ${ }^{a}$ and Nikki Stamp, MBBS, FRACS ${ }^{b}$
}

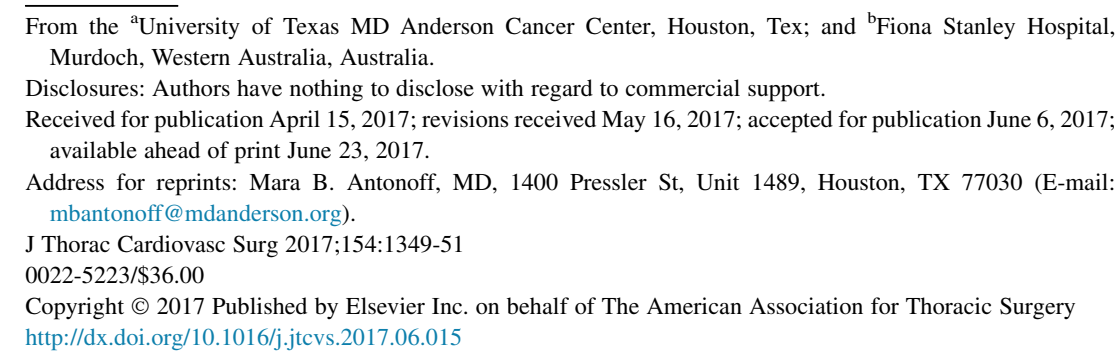

On April 3, 2017, The New Yorker released its Health, Medicine, \& Body Issue, featuring a cover art titled "Operating Theatre," a piece illustrated by the French artist Malika Favre. The image conveys the patient's perspective, looking up at 4 members of the surgical team gazing down over an operating table. In the days that followed, The New Yorker cover exploded as a source of immense social media activity. The reason for the great interest in Favre's creation: all 4 members of the surgical team were drawn as women.

After seeing the cover, Dr Susan Pitt, an endocrine surgeon at the University of Wisconsin replicated the cover art, sharing her image via Facebook, Twitter, and Instagram (Figure 1). Furthermore, she challenged other women surgeons to join her, using the hashtag, \#NYerORCoverChallenge. States Pitt, "I loved the cover and actually instantly wanted to recreate it. I'm very artistically oriented and loved the composition and beauty of the piece. I thought that it would have such a visual impact and social statement, but I didn't intend it to go viral." She goes on to explain that she was hoping to showcase women in surgery and to create a sense of pride among women surgeons. She was surprised by the results, explaining that "it wasn't until responses started rolling in that I realized how much more powerful the challenge was and how much more it meant."

In the first week since the challenge was announced, more than 1000 women sent in photographs from around the world, showcasing their own recreations of the New Yorker cover image. This social media phenomenon featuring women surgeons has caught the attention of The New Yorker itself, as well as CNN, Buzzfeed, The Washington Post, CBSNews.com, Mashable, and several additional news outlets. The story is of interest to the general public, but it's something that should be particularly noted by those of us in the surgical community. Our presence on social media is relevant, and it has an enormous impact on the ways in which surgeons are perceived.

Participation on social media by health care workers traditionally has been cautioned. Publication of sensitive

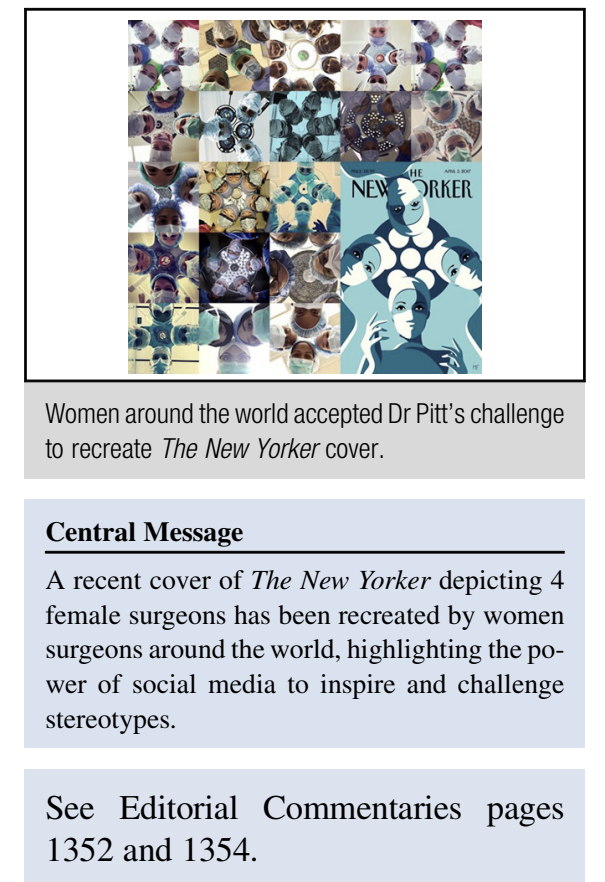

information, engaging in conduct that may be deemed "unprofessional," or being drawn into public criticism have all been cited as reasons to avoid or be sparing and cautious in the use of social media. In the last several years, however, social media is gaining popularity as a professional tool for surgeons that allows nearinstantaneous information exchange on an equitable platform. Social media is proving to have great utility in mentoring, education, networking, and academia. Most journals maintain an online presence, and the use of hashtags at meetings enhances conference experiences for attendees and observers alike. Certainly, it is of utmost importance that surgeons continue to critically assess the content which they put online for public consumption. One's public persona can be shaped in ways both positively and negatively; it's imperative that surgeons employing social media ensure that all content put forward will represent the individual, their practice, their institution, and even their specialty as a whole in a manner that is desired. Nonetheless, when executed cautiously and with the proper goals in mind, the potential impact that can be harnessed with social media is explosive.

Social media has some unique properties that make it a promising vehicle not only for networking and education, but for promoting positive and diverse role models in 


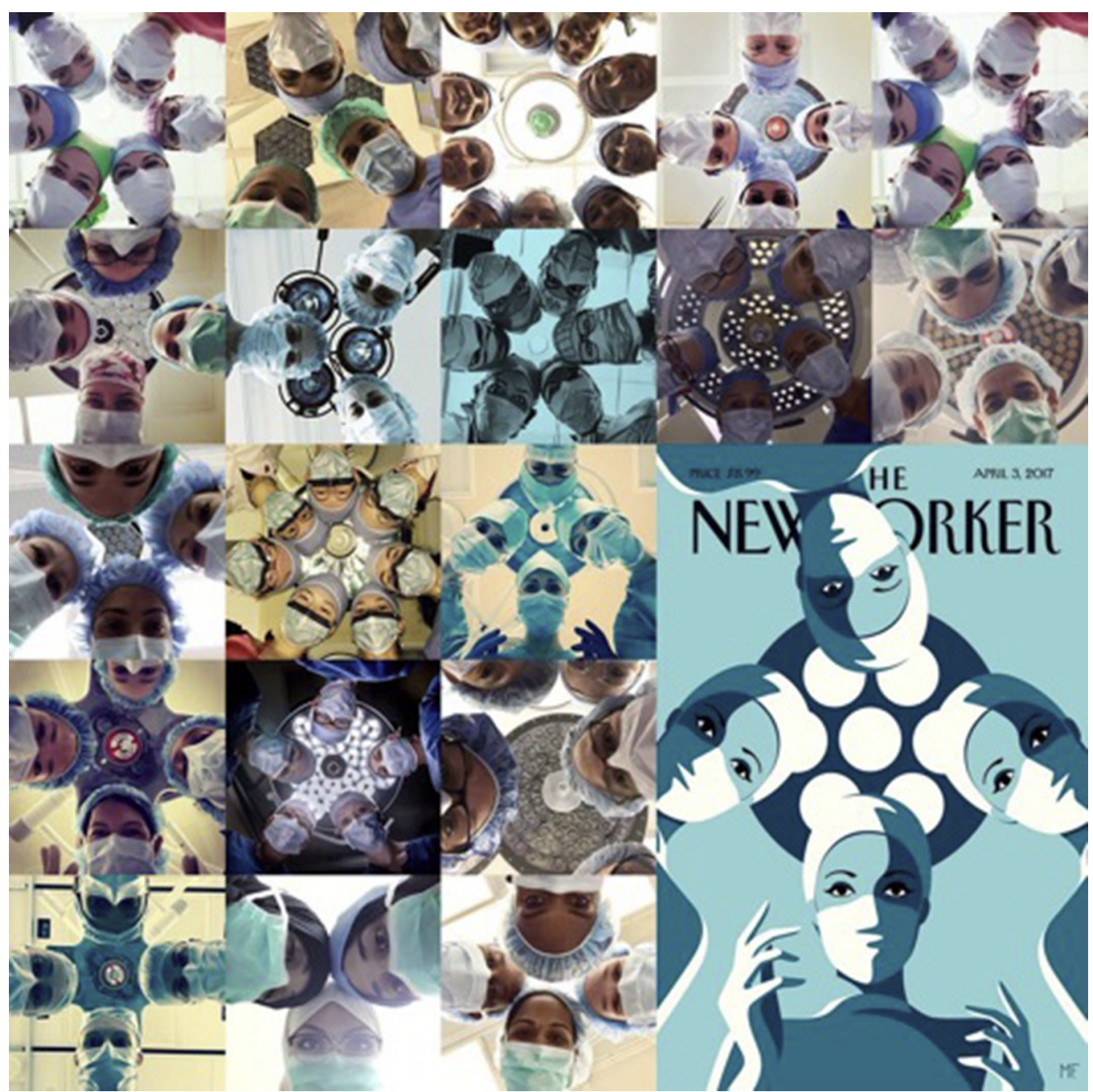

FIGURE 1. Dr Susan Pitt recreated The New Yorker cover from April 3, 2017, and thousands of women around the world accepted her challenge to participate.

surgery. ${ }^{1}$ Promotion of role models, particularly those who may not fit our cultural ideal of surgeon, enables us to shape public perceptions. Allowing the world to see that surgeons are of all genders, races, and backgrounds may inspire future generations of trainees and provide visible role models with whom diverse youth can identify.

Social media movements have been important in managing the perception of surgeons, both for the public at large as well as for potential trainees. In August 2015, general surgery resident Dr Heather Logghe coined the hashtag \#ILookLikeASurgeon after seeing a similar hashtag used in engineering. ${ }^{2,3}$ An engineer featured in a firm's advertisement received online backlash that she did not "look like an engineer," with public perception deeming her too young, female, and attractive. She responded with the hashtag \#ILookLikeAnEngineer, which was adapted by Logghe for surgeons who may not meet the public's expected appearance.
\#ILookLikeASurgeon has been used on social media millions of times since then and has been covered globally in medical journals and lay media alike. This social media phenomenon has united countless surgeons around the world, who have formed personal and professional relationships after becoming acquainted via the hashtag.

The \#NYerORCoverChallenge has brought considerable attention to the role of women as surgeons and the fact that stereotypes still exist in a world where women are increasingly present and rising through the ranks. However, as cardiothoracic surgeons, we are certainly aware that women are still drastically underrepresented. Comprising only $3.5 \%$ of diplomates ever certified by the American Board of Thoracic Surgery and 5\% of practicing cardiothoracic surgeons, women in our specialty are still a gross minority. ${ }^{4,5}$ For this reason, this most recent social media phenomenon is particularly important to women in our field. Interestingly, several women in cardiothoracic 

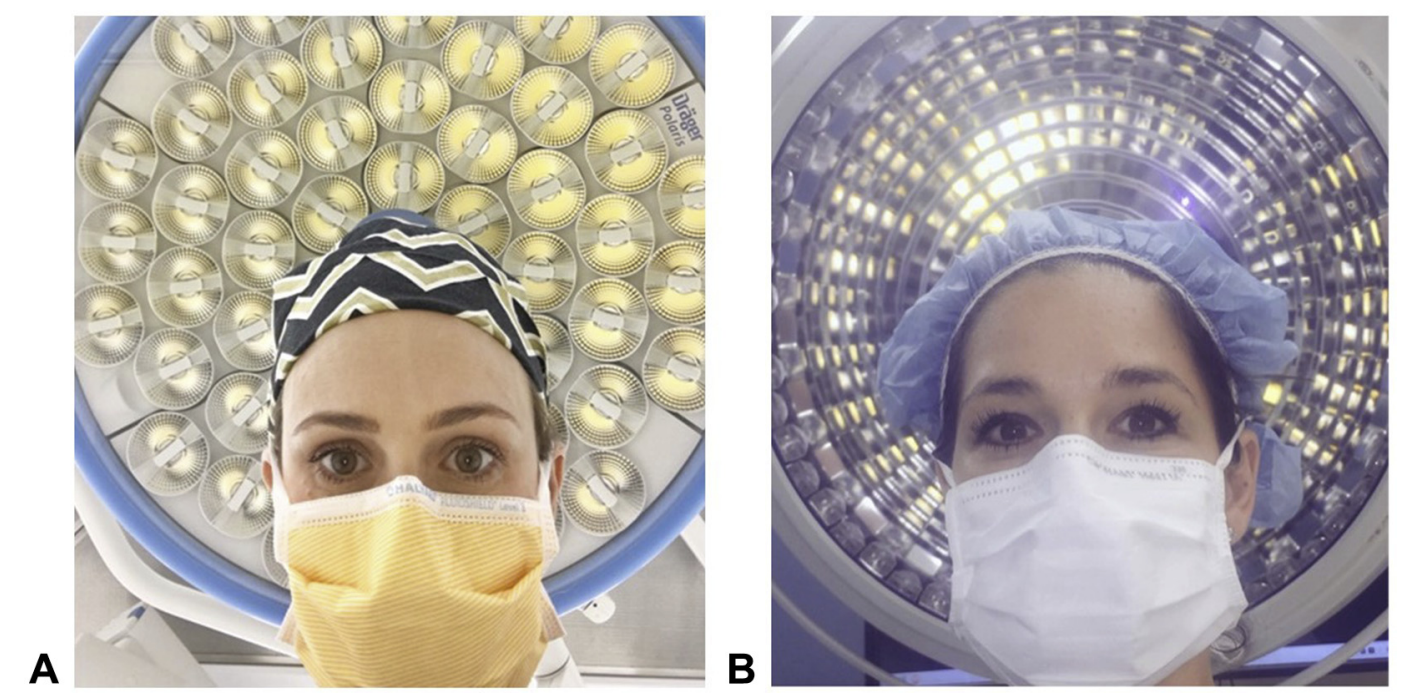

FIGURE 2. Examples of cardiothoracic surgeon recreations of The New Yorker cover. A, Dr Nikki Stamp, cardiac surgeon in Perth, Australia. B, Dr Mara Antonoff, thoracic surgeon in Houston, Texas.

surgery have recreated The New Yorker cover, showing themselves alone hovering over the operating table, symbolically bringing attention to the fact that in most of our cardiothoracic practices, we are the lone women. It is with broad networks of mutual support and the sponsorship of male colleagues that we continue to thrive, strengthening our presence as a united community of women cardiothoracic surgeons. As we want to show the best and brightest trainees that this is a specialty in which they will be challenged, welcomed, and educated, with no attention paid to their gender, we must continue to change stereotypes. By showing a new, diverse face to cardiothoracic surgery, we hope that such efforts will encourage more young women to pursue careers in our incredibly rewarding specialty. Several women thoracic surgeons have responded to the \#NYerORCoverChallenge with photos of solidarity, which have been disseminated throughout social media (Figure 2). These photographs have been compiled by CTSNet as well as the Women in Thoracic Surgery, along with Women in Thoracic Surgery-organized recreations of the cover image via group photos organized at national meetings.

Women do remain a minority in surgery and especially in cardiothoracic surgery. Social media use and campaigns such as this have the incredible power to unite female surgeons who in turn will use these connections to forge strong careers, educate others, and excel in research. We do not expect that changing the image via social media will cure all of the cultural issues that permeate our society, but we are hopeful that gradually chipping away at barriers will ultimately make a big impact. Uniting women surgeons from around the world brings the skill, experience, support, and mentorship from far and wide. We truly have the world at our fingertips and ultimately, we can deliver outstanding service to each other and our patients, buoyed by the support of all of us, who look like surgeons.

\section{References}

1. Antonoff MB. Using social media effectively in a surgical practice. J Thorac Cardiovasc Surg. 2016;151:326.

2. Hughes KA. \#ILookLikeASurgeon goes viral: how it happened. Bull Am Coll Surg. 2015;100:10.

3. Logghe HJ, Pellino G, Brady R, McCoubrey AS, Atallah S. How Twitter has connected the colorectal community. Tech Coloproctol. 2016;20:805.

4. Antonoff MB, Brown LM. Work-life balance: the female cardiothoracic surgeon's perspective. J Thorac Cardiovasc Surg. 2015;150:1422.

5. Antonoff MB, David EA, Donington JS, Colson YL, Litle VR, Lawton JS, et al. Women in thoracic surgery: 30 years of history. Ann Thorac Surg. 2016;101: 399-409. 